\title{
Innovación por Diseño y su Relación con las Variables del Entorno en una Muestra de Empresas en Bogotá - Colombia
}

\author{
César A. Bernal-Torres ${ }^{(1)^{*}}$ y Carlos E. Blanco-Valbuena ${ }^{(2)}$ \\ (1) Universidad de La Sabana, Escuela Internacional de Ciencias Económicas y Administrativas, \\ Km 7, Autopista Norte de Bogotá, Puente del Común, Campus Universitario, Chía, Colombia. \\ (e-mail: cesar.bernal@unisabana.edu.co) \\ (2) Universidad de Deusto (País Vasco - España) y Universidad Santo Tomás de Bogotá, carrera 9 n. ${ }^{\circ} 72-$ \\ 90, Bogotá, Colombia. (e-mail: carlose.co2010@gmail.com).
}

* Autor a quien debe ser enviada la correspondencia

Recibido Nov. 24, 2016; Aceptado Ene. 24, 2017; Versión final Feb. 27, 2017, Publicado Ago. 2017

\begin{abstract}
Resumen
Este artículo analiza la relación entre las variables del ambiente externo consideradas fuentes de innovación por diseño y los resultados del marketing y la rentabilidad de las empresas. Para ello se aplicó un cuestionario en la modalidad de la escala de Likert a una muestra de 327 directivos de 64 empresas medianas y grandes localizadas en Bogotá, consideradas innovadoras y con potencial para incursionar en el mercado global. Los resultados obtenidos, una vez procesada la información de los cuestionarios mediante el modelo de regresión lineal múltiple, indican que el análisis de las tendencias del mercado, del sector empresarial, de los clientes, de los desarrollos tecnológicos, y del cuidado del medio ambiente, es poco considerado para apoyar la innovación en las empresas. En los casos en que ello se hace, esas innovaciones tienen relación positiva con la imagen corporativa pero no con los resultados de las ventas, ni con la participación en el mercado ni con la rentabilidad de las empresas. Esta situación contrasta con lo que de ella se conoce por parte de los directivos de empresas en países de Europa, Norte América y Asia.
\end{abstract}

\section{Innovation by Design and its Relationship with Environmental Variables in a Sample of Companies from Bogota - Colombia}

\begin{abstract}
This article analyzes the relationship between the variables of the external environment considered sources of innovation by design and the results of marketing and profitability of companies. For this purpose questionnaire in the form of Likert scale was applied to a sample of 327 managers from 64 medium and large size companies located in Bogota (Colombia), considered to be innovative and with potential to enter the global market. Results obtained once the information of the questionnaires has been processed through a multiple lineal regression model indicate that the analysis of market tendencies, enterprise sector, technological developments, and care for the environment, is not appropriately considered to support innovation in companies. In those cases where it is taken into consideration, these innovations have a positive relationship with the corporate image, but not with sales results, market participation or with the profitability of the company. This situation contrasts with what is known by managers of companies in countries of Europe, North America and Asia.
\end{abstract}

Keywords: external environment, innovation, innovation by design, environment actors, environmental variable 


\section{INTRODUCCIÓN}

Durante la última década, especialmente en el ambiente de las organizaciones en los países desarrollados, se ha posicionado la tendencia a incorporar el diseño, desde una visión holística, como una estrategia de innovación para responder a la competencia, la globalización, la incertidumbre y el cambio a los que se ven enfrentadas las organizaciones de toda naturaleza (NWRA and the CIRCA Group Europe, 2015). Por esto, algunos gobiernos, en cooperación con los sectores empresarial y académico, han formulado políticas y programas en torno a la incorporación del diseño a la innovación (Verganti, 2013 y Kilinc et al., 2015). Para Johnson y Mchattie (2014), el diseño es una variable clave en la cultura de la innovación y por ello es importante que las organizaciones integren éste con la innovación (en sus diferentes manifestaciones), la tecnología, la administración y los clientes para construir una ventaja competitiva que se ha de manifestar de forma integral en los productos, los servicios, las comunicaciones, los sitios de trabajo, de exposición y las marcas, etcétera. En este sentido, se resalta el rol que en la actualidad tiene el pensamiento de diseño como metodología o filosofía para incorporar el diseño en las actividades organizacionales (Brown, 2009 y Beacham y Shambaugh, 2011).

Los estudios de Utterback et al. (2006) y Holladers y Van Cruysen (2009) revelan que hay fuertes relaciones entre el diseño, la innovación y el desempeño empresarial. Para estos autores, en las empresas es muy importante considerar el diseño y otras actividades no pertenecientes a la I+D como parte de un enfoque más amplio de la política de innovación. Esto sugiere que las políticas dirigidas a propiciar el diseño en el contexto empresarial tienen un efecto positivo sobre el desempeño innovador y competitivo de las empresas y del conjunto de la sociedad. En este sentido, la innovación impulsada por el diseño, desde el enfoque holístico e interdisciplinario con impacto en los ámbitos estratégico, táctico y operativo de las organizaciones en general, es una visión reciente y mucho más en las empresas de los denominados países en desarrollo.

Desde el enfoque de la innovación por diseño y donde el pensamiento de diseño juega un rol fundamental, el diseño es un factor clave para las empresas y para la competitividad de los países: no está integrado solamente en las empresas como una estrategia para impulsar la innovación y la competitividad sino que también contribuye a la competitividad nacional mediante el fomento de la cultura de la innovación en el contexto general de los países, dado que la innovación por diseño es una cuestión de sistemas que no se limita al producto, al proceso $\mathrm{o}$ al marketing para satisfacer las necesidades de los clientes sino que abarca todo el sistema empresarial, social, cultural, tecnológico y medioambiental (Norman y Verganti, 2014).

Al respecto, en el campo de las organizaciones, la innovación soportada por el diseño o innovación por diseño es una estrategia que consiste en observar y analizar las tendencias socioculturales, económicas, tecnológicas, medioambientales, etcétera, para generar innovaciones que redefinen el significado de los bienes o servicios y, en general, el conjunto de las actividades de las organizaciones. Desde esta perspectiva, el diseño tiene un enfoque holístico que permite una serie de consideraciones que van más allá de la estética tales como la funcionalidad, la ergonomía, la usabilidad, la accesibilidad, la seguridad de los bienes o los servicios, pero también de la atractividad de los sitios de trabajo y de exhibición, de la cultura organizacional y de la marca, entre otros aspectos del entorno interno empresarial. De esta forma, el diseño es deliberado y razonado dando forma y haciendo del entorno una fuente de identificación de oportunidades de innovación que no se limita sólo a satisfacer las necesidades de los clientes sino que busca que las actividades de las empresas den significado positivo a las vidas de quienes están en interacción con ella mediante sus productos, sus servicios, sus sitios de trabajo, etcétera (Norman y Verganti, 2012, Verganti, 2013 y Heskett, 2002).

Por lo antes expuesto, esta investigación tuvo como objetivo analizar la relación entre las variables del entorno empresarial consideradas fuentes de diseño y los diferentes tipos de innovación en las empresas, así como la relación entre las innovaciones soportadas por el diseño con los resultados del marketing y la rentabilidad empresarial, con el fin de aportar evidencia empírica sobre el tema para el caso colombiano, donde durante los últimos años se ha generado un interés por incorporar la innovación y, en particular, la innovación soportada en el diseño como estrategia para mejorar la capacidad competitiva de las empresas. Este estudio también puede servir de referente para contextos de países similares al mencionado interesados en este tema.

La información para el estudio se obtuvo de 327 directivos de 64 medianas y grandes empresas de sectores considerados por el Programa de Transformación Productiva (PTP) en Colombia como empresas de tallas mundiales e identificadas como innovadoras en los cinco años previos a la realización de este estudio. Esto contribuye a entender la importancia que se le da a las variables de entorno externo como las fuentes de diseño de innovaciones en estas empresas que, para el caso de Colombia, son empresas de sectores de alta potencialidad innovadora para incursionar en los mercados internacionales. 


\section{INNOVACIÓN POR DISEÑO}

Para Multu y Er (2003), el término innovación por diseño se utiliza cada vez más en el discurso académico y profesional de la administración pública y privada a partir de los años 2000. Aunque la teoría moderna de la innovación en economía ha comenzado a referirse a la relación práctica entre el diseño y la innovación y a acentuar el papel del diseño en el proceso de innovación, todavía parece insuficiente para explicar esta expansión conceptual y terminológica. La innovación por diseño tiene un potencial para convertirse en una nueva mentalidad para entender los enfoques emergentes del diseño, su contribución a la innovación y en un puente para cerrar la brecha teórica en la literatura sobre diseño, innovación y economía (Sandberg, 2011). A este respecto, Da Silva et al. (2016), al referirse a la innovación como estrategia de desarrollo integral, afirman que ésta debe ser el resultado de un proceso de educación e investigación sobre el tema.

Para Power (2004), Verganti (2009) y Potier et al. (2015), el concepto moderno de diseño, que va más allá de la estética, busca optimizar la satisfacción del consumidor, aumentar la rentabilidad, ampliar el tamaño del mercado, crear atmósferas únicas de trabajo y de relaciones con los diferentes stakeholders y trasformar la visión corporativa de las empresas. A este respecto, Lecuona (2005), Perks et al. (2005), Xu y Rickards (2007), Barrabés (2010), Gómez (2009) y Costa (2010) afirman que las empresas, en los países desarrollados, incorporan cada vez más las variables del entorno como fuentes de diseño en su estrategia para mejorar la capacidad competitiva porque han identificado que el diseño no es una actividad puntual, que hace referencia únicamente a la estética de los bienes o servicios, sino que es un proceso multidisciplinario y holístico en el que intervienen factores económicos, socioculturales, tecnológicos y medioambientales, que proporciona el conocimiento de las necesidades de los clientes y de las potencialidades de las organizaciones para responder a las exigencias del mercado cambiante, y es la fuente para la generación de innovaciones radicales en cualquiera de las actividades de las empresas. En este sentido, Hollanders y Van Cruysen (2009) consideran el diseño como parte y fuente del proceso de innovación, el cual tiene el potencial de contribuir sustancialmente a la mejora o el cambio de los bienes o servicios y de las diferentes actividades de las empresas con influencia en sus ventas y su rentabilidad. De esta forma, el diseño asume un nuevo papel en la ventaja competitiva como un elemento diferenciador vinculado a la funcionalidad, la estética, la marca de fábrica u otros intangibles (nuevo conocimiento, capital organizacional y capital relacional).

En esta misma línea de conocimiento, el Centro Danés de Diseño (2003), el Consejo de Diseño del Reino Unido (2007), el Depto. de Comercio e Industria del Reino Unido - DTI (2010) y la European Comission (2013) piensan que el diseño, desde este enfoque holístico, permite una serie de consideraciones más allá de la estética tales como la funcionalidad, la ergonomía, la usabilidad, la accesibilidad, la seguridad de los bienes o los servicios, la sostenibilidad, la marca y la cultura. Es decir, el diseño es una estrategia para mejorar la calidad de vida de las personas y producir la menor afectación posible en el planeta mediante la identificación y la solución de forma práctica, significativa y atractiva de los problemas productivos y es, además, un factor clave para la humanización de las tecnologías y los aspectos económicos de la sociedad y para dar sentido a las cosas (Norman y Verganti, 2011). A partir de los aportes de De Bevilacqua (2010) relacionados con el Centro Danés de Diseño, las experiencias sobre la innovación por diseño han generado respuestas a las necesidades de la sociedad y su evolución. Los nuevos productos representan la cultura como, por ejemplo, respetar los valores que son todavía modernos: simplificar la investigación de lo que es esencial, la inteligencia aplicada para soluciones cómodas, el esmero para lograr un entorno de calidad que apele a los sentidos y las percepciones, y la sostenibilidad de los materiales y la semántica.

De acuerdo con Norman y Verganti (2012) y Verganti (2013), aunque la mayor parte de la escritura sobre la innovación soportada en el diseño se centra en la innovación radical, caracterizada a menudo como disruptiva, las implicaciones son claras tanto en las innovaciones incrementales (relacionadas con mejoras significativas en las ya existentes) como en las radicales (producir o hacer algo que no existía). Para estos autores, las innovaciones radicales en el conjunto de la sociedad son poco frecuentes y para su éxito se requieren mejoras o adaptaciones continuas, indicando con ello que las innovaciones incrementales suelen estar siempre presentes en toda innovación y, por ende, el diseño ha de estar tanto en la una como en la otra. Además, agregan que la innovación incremental es necesaria para transformar la radical en una forma que sea aceptable para aquellos que están más allá de los primeros adoptantes y concluyen que, sin innovación radical, la innovación incremental alcanza el límite y, sin ésta, no se capta el potencial ofrecido por el cambio radical. De otra parte, para el caso de los países en desarrollo existe generalizado consenso en que la acumulación de cambios menores y pequeñas innovaciones tiene tanto impacto en el producto o en los procesos como el que tiene la innovación radical (Jaramillo et al., 2000).

De acuerdo con Verganti (2010a), desde el punto de vista estratégico, en la actualidad existen básicamente dos enfoques que analizan el rol del diseño en la dinámica empresarial. El primero es el denominado diseño centrado en el usuario y que considera que el desarrollo de los bienes o servicios siempre debe comenzar 
desde un análisis detallado de las necesidades de los usuarios (Chayutsahakij y Poggenpohl, 2002, Lojacono y Zaccai, 2004, Ulrich, 2004 y Veryzer y Borja de Mozota, 2005). El diseño debe ser una cultura orientada a la construcción sostenida de experiencias constructivas para el cliente o usuario de un bien o un servicio y las empresas decididas a apostar por él deben volver constantemente hacia la experiencia de sus clientes y diseñar y diseñarse en función de ella (Kumar y Whitney, 2003, Rosenthal y Capper, 2006, Selden, 2006, y Kilinc et al., 2015).

Según Leonard-Barton (2005), las razones por las cuales los usuarios se implican en el desarrollo de un nuevo sistema técnico están enfocadas en dos planos. El primero se relaciona con el proceso de implantación que implica un cierto nivel de cambio en el trabajo. El segundo tiene que ver con la implicación de los usuarios en el diseño de las herramientas, que dan lugar a mejores productos porque los usuarios tienen conocimientos especializados sobre el entorno en el que van a utilizar estas herramientas. Es indudable que estos conocimientos deben incorporarse al proceso de diseño. Para Brunner y Emery (2008), desde este enfoque, el diseño no consiste tan sólo en generar un bien o un servicio atractivo sino en cuidar cada aspecto de la experiencia del cliente, significa poner atención al producto, a las tiendas, a las páginas web o al servicio al cliente y, en general, a la forma de pensar y de actuar de la empresa para construir una relación en la que el cliente perciba una identificación emocional con el bien o servicio y con la propia empresa.

Desde este enfoque, el pensamiento de diseño (design thinking, en inglés) tiene un rol preponderante al constituirse en una metodología de innovación centrada en la persona que consiste en aprovechar la sensibilidad del diseñador y sus métodos para la solución de problemas relacionados con la satisfacción de las necesidades de los clientes por parte de las empresas de modo tecnológicamente factible y comercialmente viable (Brown, 2009). Esto se da porque el pensamiento de diseño prevalece en el proceso de diseño, más que en el resultado final, gracias a que esta metodología integra conocimientos técnicos del diseño, conocimientos del campo de las ciencias sociales, conocimiento específico de la empresa y la ingeniería y conocimiento de los clientes y su situación problema mediante el trabajo activo de equipos multidisciplinares.

El segundo enfoque sobre el diseño como estrategia empresarial, citado por Verganti (2010a), es el de innovación por diseño o innovación impulsada por el diseño, que consiste en generar innovaciones que redefinen el significado de los bienes o servicios y, en general, el conjunto de las actividades de las organizaciones (NWRA and the CIRCA Group Europe, 2015). Es decir, son innovaciones que aportan nuevos significados de los bienes y servicios y de la propia actividad empresarial (Verganti, 2010a, Sandberg, 2011, Norman y Verganti, 2012, y Verganti, 2013). Este enfoque no parte de las ideas o las necesidades de los usuarios porque considera que el contexto sociocultural en el que están inmersos actualmente los usuarios los hace proclives a interpretaciones que están en línea con lo que está sucediendo y con lo conocido hasta el momento, razón por la cual son poco propensos a aportar a la generación de innovaciones, especialmente de las denominadas radicales (Norman y Verganti, 2014). Ejemplos de innovaciones sin un análisis de las necesidades de los clientes son los más comunes: la iluminación eléctrica en los hogares, el automóvil y el avión, la radio y la televisión, el Facebook y el Twitter, las cuales se han producido simplemente porque sus inventores pensaron que eran interesantes.

Desde este enfoque, los cambios en los significados implican también cambios en los modelos socioculturales, algo que sólo puede ser entendido observando y analizando los fenómenos y las prácticas socioculturales a largo plazo con una perspectiva futurista amplia. Así, la innovación se inicia a partir de la comprensión de las tendencias de las dinámicas explicitas y tácitas de los modelos socioculturales mediante propuestas nuevas con significados y lenguajes que a menudo implican un cambio en los regímenes socioculturales (Norman y Verganti, 2012). Para este enfoque, sin dejar de analizar las necesidades del usuario, se analiza el conjunto de actores del entorno general de la empresa tales como las tendencias tecnológicas, otras industrias, las ideas de los artistas, los centros culturales, los diseñadores, las universidades y las exhibiciones, pero, en particular, los cambios en las tendencias de las dinámicas socioculturales que crean nuevos significados para dar sentido a las cosas y que facilitan y propician la innovación en los bienes y servicios y el propio rediseño de las organizaciones o sus formas de ser (Verganti, 2010b y Norman y Verganti, 2012).

Según Espinosa et al. (2008), la innovación acompañada por el diseño es un proceso de aprendizaje en el cual se generan flujos de conocimientos, competencias y capacidades, los cuales hacen parte del capital organizacional que hace que la empresa pueda competir mejor en el mercado, todo ello teniendo en cuenta las variables organizacionales. En este sentido, de acuerdo con Norman y Verganti (2012) y Verganti y Öberg (2012), la innovación basada en el diseño no se soporta en herramientas o métodos peculiares de creatividad sino en el conocimiento de las tendencias de los modelos socioculturales mediante el análisis de intérpretes claves. Se trata de un proceso de investigación colectiva y en red sobre los significados y 
lenguajes de diseño, que tiene lugar fuera de los límites de la empresa, que permite anticipar e influir en la aparición de nuevos significados para los usuarios y la propia sociedad de los productos y de la misma dinámica empresarial con capacidad de influir en los cambios de los regímenes socioculturales del conjunto de la sociedad (Soh y Roberts, 2003, Sorenson y Waguespack, 2005, y Dow et al., 2010). Ejemplos clásicos de estas innovaciones (de productos) son los relojes Swatch, que cambiaron el concepto del reloj como aparato para medir el tiempo a reloj como parte de las prendas de vestir; el huevo kínder que trasformó el concepto de golosina a entretenimiento; el teléfono celular que cambio el concepto de teléfono para convertirse en dispositivo móvil. Innovaciones estas que una vez introducidas en la sociedad, gracias a la participación de diversos agentes de la sociedad o mediante el análisis de variables del entorno, han venido siendo mejoradas para impactar de mejor forma en el conjunto de la sociedad.

Desde esta perspectiva de la innovación impulsada por el diseño, una nueva economía, natural y social, en red, emerge con una serie de implicaciones que alcanza todos los aspectos de la actividad humana, en donde el diseño juega un papel fundamental en lo que tiene que ver con el cambio de la cultura tradicional y la manera de actuar de las empresas y de la sociedad (Manzini, 2010, Consejo Europeo de Liderazgo de Diseño, 2012, y Norman y Verganti, 2014). Por este motivo y dado que, en general, el diseño en los países en desarrollo, especialmente de América Latina, sigue siendo entendido desde una concepción clásica diseño como sinónimo de estética-, su incorporación a la dinámica de las organizaciones es muy ocasional y casi nula en algunos países (Rodríguez y Rodríguez, 2007). Este estudio se orientó a responder los siguientes tres objetivos específicos: a) evaluar la relación directa entre las variables del entorno externo tales como las tendencias del mercado, las expectativas y necesidades de los clientes, la dinámica de la competencia, la protección del medio ambiente, las tendencias del sector, las sugerencias de los proveedores, las visitas a ferias y otros eventos y la existencia de departamentos de diseño con la innovación (de productos, de proceso, de marketing y organizacionales) en las empresas; b) evaluar la relación directa entre los actores económicos y sociales del entorno tales como los analistas socioeconómicos, los medios de comunicación, las instituciones académicas, los artistas, los centros culturales, etcétera, con la innovación de las empresas; y c) evaluar la relación directa entre la innovación por diseño y los resultados de la actividad empresarial tales como las ventas, la participación en el mercado, la rentabilidad y la imagen corporativa.

\section{METODOLOGÍA}

Para dar respuesta a los objetivos antes descritos se obtuvo la información de 327 directivos (uno por cada área: gerencia general o vicepresidencia, técnica o de producción, marketing o comercial, financiera y de desarrollo humano) de 64 medianas y grandes empresas de los sectores considerados en Colombia por el Programa de Transformación Productiva (PTP) como sectores de talla mundial: autopartes (4), metalmecánica (5), plásticos (5), industria gráfica (6), textiles, confecciones, diseño y moda (6), cuero y calzado (6), cosméticos y artículos de aseo personal (5), software y servicios de TI (5), turismo de salud (4), energía eléctrica, bienes y servicios conexos (5), PO\&O (productos de tercerización a distancia) (5), hortofrutícola (4), lácteos (5) y chocolatería (5), localizadas en la ciudad de Bogotá, caracterizadas por contar con más de diez años continuos de actividad y que durante los cinco años previos al estudio fueron consideradas como innovadoras. El número de empresas por sector que participaron del estudio obedeció a la relevancia o reconocimiento de las empresas en su sector en lo referente a innovación y a su disposición a participar del estudio, no a su representatividad estadística. En este sentido, de un total de 100 empresas invitadas, finalmente 64 aceptaron participar.

La información se obtuvo mediante la aplicación de un cuestionario con estructura de escala Likert, diseñado a partir de los planteamientos de Gómez (2009), Costa (2010), Verganti (2010b) y Norman y Verganti (2012), quienes destacan la importancia de que las empresas interactúen con los diversos agentes o actores del entorno y analicen variables como las expectativas de los clientes, las tendencias del mercado y del sector de actividad, las tendencias tecnológicas, medioambientales y socioculturales, etcétera, para soportar la innovación en su diferentes modalidades (de producto, de proceso, de marketing, organizacional, etcétera), la que, a su vez, soportada en el diseño, crea valor para la propia empresa y para el conjunto de la sociedad. Previa a la aplicación, el cuestionario fue validado por un panel de tres expertos y mediante el coeficiente Alfa de Cronbach, el cual se estimó con la siguiente fórmula:

$\alpha=\frac{K}{K-1}\left[1-\left(\frac{\sum V_{i}}{V t}\right)\right]$

Donde: $K=$ Número de ítemes, $V_{i}=$ Varianza de cada ítem y $V_{t}=$ Varianza total

El valor obtenido del índice de consistencia interna fue $\propto=0.937$, lo que indica que el instrumento utilizado es fiable. El cuestionario fue diligenciado durante el período de marzo a noviembre de 2014, de forma personal, en las instalaciones de cada empresa participante, en interacción conjunta entre los 
investigadores y los directivos empresariales y con apoyo de un grupo de estudiantes de último semestre del Programa de Administración de Empresas de la Escuela Internacional de Ciencias Económicas y Administrativas de la Universidad de La Sabana. Para identificar la relación o la incidencia de las variables objeto del estudio, se utilizó el modelo de regresión lineal múltiple de la forma:

$\widehat{Y}=\beta_{0}+\beta_{1} * X_{1}+\beta_{2} * X_{2}+\beta_{3} * X_{3}+\cdots+\beta_{\mathrm{k}} * X_{\mathrm{k}}$

Donde: $\widehat{Y}:$ Variable dependiente = tipo de innovación (de producto, de proceso, de marketing $\mathrm{y}$ organizacional); $\beta_{\mathrm{i}} * \mathrm{X}_{\mathrm{i}}$ : Variable independiente $(\mathrm{i}-$ ésima $)=$ variables del entorno

(i) Tendencias del mercado: grado de conocimiento que las personas de la empresa tienen de los cambios o los patrones que caracterizarán el mercado en los próximos años; (ii) Expectativas de los clientes: grado de conocimiento que las personas de la empresa tienen de lo que esperan los clientes de la empresa y de sus productos (bienes o servicios); (iii) Dinámica de la competencia: grado de conocimiento que las personas de la empresa tienen de los planes de las empresas de su sector de actividad; (iv) Protección del medio ambiente: grado de conocimiento y compromiso que las personas de la empresa tienen para proteger el medioambiente; $(v)$ Tendencias del sector: grado de conocimiento que las personas de la empresa tienen sobre los cambios que se están dando y se darán en los próximos años en las empresas del sector; (vi) Sugerencias de los proveedores: grado de recepción que las personas de la empresa tienen sobre las recomendaciones que hacen sus proveedores respecto a diferentes actividades del mercado; (vii) Visitas a ferias y otros eventos: frecuencia con que las personas de la empresa asisten a ferias y demás eventos relacionados con su sector, actividad económica y tecnología; (viii) Empleo de expertos en diseño: grado en que la empresa se soporta en expertos en diseño para realizar sus diferentes actividades; (iv) Existencia del departamento de diseño: grado en que la empresa dispone y usa el departamento de diseño para el desarrollo de sus actividades.

$\beta_{0}$ : Término independiente del modelo

Para el ajuste de los modelos propuestos se utilizó la metodología de mínimos cuadrados. El nivel de confianza $(Z)$ utilizado para la aprobación o rechazo de las hipótesis fue 95\%.

$\widehat{\beta}=\left(X^{\prime} X\right)^{-1} * X^{\prime} Y$

Donde: $\widehat{\beta}$ : Vector de coeficientes, X : Matriz de observaciones independientes (variables del entorno) $\mathrm{Y}:$ Vector de observaciones dependientes

\section{RESULTADOS Y DISCUSIÓN}

Los resultados de este estudio se analizan en cuatro dimensiones: a) resultados relacionados con la generación de cada uno de los tipos de innovación (productos, procesos, marketing y organizacional) en las empresas, b) resultados de la relación entre las variables del entorno con la innovación y el diseño en las empresas, c) relaciones entre las tendencias de las variables socioculturales (actores) y la innovación en las empresas, y d) relación entre la innovación soportada en el diseño y los resultados de la actividad empresarial.

\section{Innovación en las empresas}

En general, los resultados del estudio indican que el 89,7\% de los directivos entrevistados están de acuerdo en que la innovación es una variable estratégica para mejorar la competitividad empresarial. En ese sentido, el $74,2 \%$ de ellos manifestaron que, en las empresas en donde ellos trabajan, actualmente se realizan cambios o mejoras significativas en los productos o servicios existentes, el $67,9 \%$ que se realizan cambios 0 mejoras en los procesos de producción, el 65,9\% que se realizan cambios o mejoras en los procesos administrativos, el 56,7\% que se realizan cambios o mejoras en los procesos de mercadeo y comunicación de los productos o servicios que ofrece la empresa y el $51,6 \%$ que se realizan mejoras o cambios orientados a la protección del medio ambiente.

Considerados de forma agregada, los datos indican que las empresas participantes del estudio confirman ser innovadoras según el criterio por el cual se les seleccionó, sin embargo, cuando se preguntó por la cantidad de nuevos bienes o servicios que anualmente las empresas llevan al mercado, sólo el 23,2\% de ellas manifestaron generar más de dos nuevos bienes o servicios. Los resultados también indican que, en general, los directivos conservan la visión tradicional de hacer énfasis en la dinámica y los cambios en las empresas a partir de planes originados por las experiencias internas más que por la dinámica del entorno 
empresarial. Esto porque el 73,5\% de los directivos afirman que los cambios o mejoras mencionados son una respuesta a los planes propios de la empresa, el 69,1\% a las condiciones de la competencia, el 66,7\% a las tendencias del mercado donde opera la empresa, el 62,5\% a las expectativas y las necesidades de los clientes, el 46,3\% a las sugerencias de los proveedores, el 41,9\% a las exigencias para la protección del medio ambiente y el $33,5 \%$ a visitas a ferias, congresos u otros eventos relacionados con las actividades de las empresas.

En lo referente a la importancia del diseño y en particular a la innovación por diseño como estrategia para generar ventaja competitiva para las empresas, el 79,8\% de los directivos consideran que en las empresas sí se emplea el diseño para las actividades de marketing, el 73,1\% para realizar los cambios o las mejoras en sus bienes o servicios, el 61,9\% para mejorar la imagen interna y externa de la empresa y el 43,5\% para mejorar las condiciones de trabajo. Así, el 83,8\% de ellos dicen que la empresa para sus cambios se apoya en profesionales de diseño y el 49,2\% en departamentos propios de diseño. Cuando se preguntó si las innovaciones generadas en las empresas se hacen: a) para contribuir a cambiar el significado de las cosas o b) contribuir a mejorar la calidad de vida de alguno o varios de sus grupos de interés, sólo el $11.7 \%$ y el $24,7 \%$ respectivamente, de los participantes puntuó valores de 4 y 5 en una escala de 1 a 5 , donde 1 indica totalmente en desacuerdo y 5 totalmente de acuerdo.

Las razones expuestas por los directivos respecto a la limitada utilización del diseño como base de la innovación en las empresas son: el desconocimiento del nuevo significado y de la importancia del diseño en la innovación $(89,7 \%)$, los altos costos del diseño $(87,4 \%)$, la idea de que los profesionales del diseño no responden a las necesidades de la empresa (76,7\%), la no asignación de presupuesto para actividades de diseño $(68,2 \%)$ y la falta de cultura en la empresa por el uso de diseño $(67,7 \%)$. Esto puede estar indicando que, en general, la gerencia en las empresas mantiene el concepto clásico de diseño como sinónimo de estética y desconoce el actual concepto amplio de diseño y, en particular, el de innovación por diseño (Norman y Verganti, 2012).

En cuanto a la importancia del análisis de las variables socioculturales y de sus tendencias como una estrategia para la generación de innovación basada en el diseño, los resultados indican que sólo el 27,4\% de los directivos manifestaron tener en cuenta los análisis de las tendencias socioculturales, el $23,9 \%$ los de los medios de comunicación, el $20,6 \%$ los de las instituciones académicas, el $20,1 \%$ las tendencias tecnológicas, el $17,3 \%$ los de los centros culturales, el $16,7 \%$ las ideas de los artistas, el $11,4 \%$ los de prospectiva y el $7,4 \%$ los contactos con otras industrias. Situación contraria a la que muestran los resultados de los estudios realizados por Manzini (2010) y Norman y Verganti (2012) en empresas de los países desarrollados. No obstante lo antes mencionado, los gerentes reconocen el rol positivo de la innovación soportada en el diseño en los resultados de la actividad empresarial. Esto en razón de que el $66,7 \%$ de los directivos consideran que, como consecuencia de la innovación realizada con apoyo del diseño, se incrementaron las ventas, el 64,0\% que se mantuvo o se ganó participación en el mercado, el 55,3\% que se mejoró la rentabilidad de la empresa y el $66.4 \%$ que se mejoró la imagen de la empresa.

\section{Relación entre las variables del entorno y la innovación empresarial}

Los datos de la tabla 1 y de la ecuación (4) indican que, con un nivel de confianza del 95\%, las ideas provenientes de los clientes, la dinámica de la competencia, las tendencias del sector, las políticas de protección del medio ambiente, las visitas a ferias y otros eventos relacionados con la actividad de la empresa y la existencia de departamentos de diseño, tienen relación directa con la innovación de productos en las empresas, pero variables como las tendencias del mercado, las sugerencias de los proveedores y el empleo de expertos en diseño, no presentan relación. Esto revela que aunque para la innovación en los productos las empresas aprovechan la información del entorno, esa información se limita a sólo algunos agentes clave, desaprovechado la de otros igualmente claves como los proveedores, las tendencias del mercado y los profesionales del diseño, resultados estos que contrastan con los encontrados en los estudios realizados por Norman y Verganti (2012) y Verganti y Öberg (2012).

$\widehat{Y}=0.50000152+0.06655393 * X_{1}+0.80319438 * X_{2}-0.1540253 * X_{3}-0.1012805 * X_{4}+0.5394383 *$

$X_{5}+0.004569 X_{6}+0.16819358 * X_{7}-0.109327 * X_{8}+0.27490239 * X_{9}$

Para el caso de las innovaciones de proceso, la tabla 2 y la ecuación (5) indican que, de las variables del entorno, la dinámica de la competencia, las políticas de protección del medio ambiente y la existencia de departamentos de diseño afectan de forma directa esas innovaciones. Esto significa que las fuentes de ideas que utilizan las empresas para este tipo de innovaciones son muy restringidas, situación que puede obedecer al desconocimiento, por parte de la gerencia, de la diversidad de fuentes existentes en el entorno que contribuyen a los cambios o mejoras en sus procesos productivos (Manzini, 2010, Consejo Europeo de Liderazgo de Diseño, 2012, y Norman y Verganti, 2014). 
$\widehat{Y}=1.66256638+0.09397648 * X_{1}+0.0853861 * X_{2}+0.41149672 * X_{3}+0.12259541 * X_{4}+0.0762783 *$

$X_{5}+0.06514864 * X_{6}+0.29645941 * X_{7}+0.03037327 * X_{8}+0.13495563{ }^{*} X_{9}$

Tabla 1: Relación entre variables del entorno y la innovación de productos

\begin{tabular}{lrrr}
\hline Variables del entorno $\left(\mathrm{X}_{\mathrm{J}}\right)$ & Coeficientes $/ \beta_{(i)}$ & Estadístico $t$ & Probabilidad / $p$ \\
\hline Intercepción & 0.50000152 & 2.04406176 & 0.04198704 \\
Tendencias del mercado & 0.06655393 & 1.03734431 & 0.30056977 \\
Expectativas de los clientes & 0.80319438 & 10.5839483 & $6.7244 \mathrm{E}-22$ \\
Dinámica de la competencia & -0.1540253 & -2.4703153 & 0.01416219 \\
Protección del medio ambiente & -0.1012805 & -2.35905 & 0.01908548 \\
Observar las tendencias del sector & 0.5394383 & 8.54385911 & $1.2559 \mathrm{E}-15$ \\
Sugerencias de los proveedores & 0.004569 & 0.0898931 & 0.92844355 \\
Visitas a ferias y otros eventos & 0.16819358 & 2.43791168 & 0.01545608 \\
Empleo de expertos en diseño & -0.109327 & -1.620395 & 0.10638338 \\
Existencia del departamento de diseño & 0.27490239 & 4.41760012 & $1.4763 \mathrm{E}-05$ \\
\hline
\end{tabular}

Tabla 2: Relación entre variables del entorno y la innovación en los procesos

\begin{tabular}{lrrr}
\hline Variables del entorno $\left(\mathrm{X}_{\mathrm{J}}\right)$ & Coeficientes $/ \beta_{(i)}$ & Estadístico $t$ & \multicolumn{1}{c}{ Probabilidad / $p$} \\
\hline Intercepción & 1.66256638 & 4.83703375 & $2.2964 \mathrm{E}-06$ \\
Tendencias del mercado & 0.09397648 & 1.04242737 & 0.29821259 \\
Expectativas de los clientes & 0.0853861 & 0.8007391 & 0.424037 \\
Dinámica de la competencia & 0.41149672 & 4.69682184 & $4.346 \mathrm{E}-06$ \\
Protección del medio ambiente & 0.12259541 & 2.03218345 & 0.04318394 \\
Observar las tendencias del sector & 0.0762783 & 0.9907801 & 0.32274334 \\
Sugerencias de los proveedores & 0.06514864 & 1.05118237 & 0.29418181 \\
Visitas a ferias y otros eventos & 0.29645941 & 4.95370572 & $1.336 \mathrm{E}-06$ \\
Empleo de expertos en diseño & 0.03037327 & 0.47870352 & 0.63255976 \\
Existencia del departamento de diseño & 0.13495563 & 2.30611325 & 0.02190685 \\
\hline
\end{tabular}

En lo referente a las innovaciones organizacionales, los datos de la tabla 3 y la ecuación (6) indican que su relación directa está con las tendencias del mercado, la competencia y las políticas de protección del medio ambiente, pero no con las expectativas y necesidades de los clientes, ni con las tendencias del sector, ni con la visita a ferias o congresos de actividades relacionadas con la actividad empresarial ni con el campo del diseño. Esto indica que las innovaciones realizadas por las empresas requieren una orientación más amplia para que impacten en los diferentes ámbitos de las empresas, tal como lo plantean el Centro Danés de Diseño (2003), el Consejo de Diseño del Reino Unido (2007), el Departamento de Comercio e Industria del Reino Unido - DTI (2010) y la European Comission (2013) para las empresas en Europa.

$\hat{Y}=0.28750004+0.46273825 * X_{1}-0.0910723 * X_{2}+0.216001 * X_{3}+0.10452906 * X_{4}-0.0582205 *$

$X_{5}+0.39145448 * X_{6}+0.09036576 * X_{7}+0.01045571 * X_{8}+0.09134449{ }^{*} X_{9}$

La tabla 4 y la ecuación (7) muestran que las innovaciones en el campo del marketing tienen relación directa con las necesidades y expectativas de los clientes, la dinámica de la competencia, las normas de protección del medio ambiente, las sugerencias de los proveedores y, en particular, con el empleo de diseñadores y la existencia de departamentos de diseño en las empresas, pero no con las exigencias del mercado, ni con las tendencias del sector, ni con la asistencia a ferias o demás eventos relacionados con la actividad de las empresas ni con el diseño, situación ésta que igualmente restringe la capacidad innovadora de las empresas.

$\widehat{Y}=-0.1409066-0.0772485 * X_{1}+0.26684102 * X_{2}+0.2608602 * X_{3}+0.35191875 * X_{4}-0.0582205 *$

$X_{5}+0.39145448 * X_{6}+0.09036576 * X_{7}+0.06549007 * X_{8}+0.27126163{ }^{*} X_{9}$ 
Tabla 3: Relación entre variables del entorno y la innovación organizacional

\begin{tabular}{lrrr}
\hline Variables del entorno $\left(\mathrm{X}_{\mathrm{J}}\right)$ & Coeficientes $/ \beta_{(i)}$ & Estadístico $t$ & Probabilidad $/ p$ \\
\hline Intercepción & 0.28750004 & 1.24267364 & 0.21514318 \\
Tendencias del mercado & 0.46273825 & 7.62572408 & $4.9582 \mathrm{E}-13$ \\
Expectativas de los clientes & -0.0910723 & -1.2688483 & 0.20566593 \\
Dinámica de la competencia & 0.216001 & 3.66279426 & 0.00030404 \\
Protección del medio ambiente & 0.10452906 & 2.57421493 & 0.01061883 \\
Observar las tendencias del sector & -0.0582205 & -0.8136641 & 0.41660553 \\
Sugerencias de los proveedores & 0.39145448 & 6.79590342 & $0.7324 \mathrm{E}-11$ \\
Visitas a ferias y otros eventos & 0.09036576 & 1.62465857 & 0.10548537 \\
Empleo de expertos en diseño & 0.01045571 & 0.17770369 & 0.85909677 \\
Existencia del departamento de diseño & 0.09134449 & 1.6832153 & 0.09355725 \\
\hline
\end{tabular}

Tabla 4: Relación entre variables del entorno y la innovación en marketing

\begin{tabular}{lrrr}
\hline Variables del entorno $\left(\mathrm{X}_{\mathrm{J}}\right)$ & Coeficientes $/ \beta_{(i)}$ & \multicolumn{1}{c}{ Estadístico $t$} & Probabilidad $/ p$ \\
\hline Intercepción & -0.1409066 & -0.4303189 & 0.66733149 \\
Tendencias del mercado & -0.0772485 & -0.8994468 & 0.36927341 \\
Expectativas de los clientes & 0.26684102 & 2.62673378 & 0.00914903 \\
Dinámica de la competencia & 0.2608602 & 3.12539214 & 0.00198368 \\
Protección del medio ambiente & 0.35191875 & 6.12336426 & $3.4946 \mathrm{E}-09$ \\
Observar las tendencias del sector & -0.0582205 & -0.8136641 & 0.41660553 \\
Sugerencia de los proveedores & 0.39145448 & 6.79590342 & $7.7324 \mathrm{E}-11$ \\
Visitas a ferias y otros eventos & 0.09036576 & 1.62465857 & 0.10548537 \\
Empleo de expertos en diseño & 0.06549007 & 1.01192357 & 0.03125338 \\
Existencia del departamento de diseño & 0.27126163 & 4.54438577 & $0.0126 \mathrm{E}-06$ \\
\hline
\end{tabular}

\section{Relación entre actores de la dinámica económica-social y la innovación}

La tabla 5 y la ecuación (8) indican que, en general, con un nivel de confianza del 95\%, en lo referente a la innovación soportada en el diseño, es decir, la innovación radical capaz de generar nuevos significados en los bienes o los servicios y en el de las demás actividades empresariales, su relación directa está sólo con las tendencias tecnológicas y con los contactos directos con las instituciones académicas, lo cual significa que las empresas desaprovechan la diversidad de fuentes externas relacionadas con la dinámica sociocultural que ofrece excelentes oportunidades para la generación de innovaciones.

$\hat{Y}=-0.1867017-0.099933 * X_{1}+0.02482606 * X_{2}+0.07873918 * X_{3}+0.43750206 * X_{4}+0.10797603 *$

$X_{5}+0.02243104 * X_{6}+0.08079985 * X_{7}+0.16053615 * X_{8}$

Tabla 5: Relación entre actores económico-sociales y la innovación basada en el diseño

\begin{tabular}{lrrr}
\hline Tendencias de la dinámica social $\left(\mathrm{X}_{\mathrm{J}}\right)$ & Coeficientes $/ \beta_{(i)}$ & Estadístico $t$ & Probabilidad $/ p$ \\
\hline Intercepción & -0.1867017 & -0.5837232 & 0.55992867 \\
Analistas económico-sociales & -0.099933 & -1.6733978 & 0.09548983 \\
Medios de comunicación & 0.02482606 & 0.43801765 & 0.66174871 \\
Prospectivistas & 0.07873918 & 1.36944598 & 0.17207905 \\
Instituciones académicas & 0.43750206 & 6.34206523 & $1.0423 \mathrm{E}-09$ \\
Artistas & 0.10797603 & 1.56842943 & 0.11803573 \\
Centros culturales & 0.02243104 & 0.39429899 & 0.69369378 \\
Otras industrias & 0.08079985 & 0.86332952 & 0.38877724 \\
Tendencias tecnológicas & 0.16053615 & 2.20661984 & 0.02824283 \\
\hline
\end{tabular}




\section{Relación entre la innovación por diseño y los resultados de la empresa}

Finalmente, la tabla 6 y la ecuación (9) indican que, con un nivel de confianza del 95\%, la innovación soportada en el diseño sólo tiene relación directa con los aportes del diseño a la imagen corporativa pero no con los resultados de las ventas, ni con la participación en el mercado ni con la rentabilidad de las empresas, resultados que contrastan con los encontrados en los estudios realizados sobre el tema en empresas de países europeos, asiáticos y norteamericanos (Centro Danés de Diseño, 2003, Perks et al., 2005, Xu y Rickards, 2007, Goritti, 2010, y Verganti y Öberg 2012).

$$
\widehat{Y}=2.80130476+0.05867397 * X_{1}-0.0235274 * X_{2}+0.0870102 * X_{3}+0.16819358 * X_{4}
$$

Tabla 6: Relación entre el diseño y los resultados de la actividad empresarial

\begin{tabular}{lrrr}
\hline Resultados de la actividad empresarial & Coeficientes / $\beta_{(i)}$ & Estadístico $t$ & Probabilidad / $p$ \\
\hline Intercepción & 2.80130476 & 13.257954 & $7.2048 \mathrm{E}-31$ \\
Ventas de la empresa & 0.05867397 & 0.77899967 & 0.43670222 \\
Participación en el mercado & -0.0235274 & -0.3709285 & 0.71099878 \\
Rentabilidad & 0.0870102 & 1.55085562 & 0.12217647 \\
Imagen corporativa & 0.16819358 & 2.43791168 & 0.01545608 \\
\hline
\end{tabular}

\section{DISCUSIÓN FINAL}

Los datos referidos en este artículo ponen en evidencia que es escaso el conocimiento y el interés particular por la innovación basada en el diseño como una estrategia capaz de generar ventaja competitiva sostenible y que los directivos tienen poca información sobre el verdadero significado de este tipo de innovación y, en particular, de los aportes del diseño a la competitividad, situación que contrasta con lo que de ella se conoce por parte de los directivos de empresas de países desarrollados. Los resultados del estudio también indican que debido a la escasa importancia que en las empresas se da al diseño como estrategia de innovación, ya sea centrada en el usuario o propiamente impulsada por el diseño, son muy limitados o casi nulos los beneficios relacionados con los cambios o mejoras en los bienes o servicios así como en los procesos de solución de problemas de la organización, en el proceso de toma de decisiones, en el logro de mayor eficacia organizacional, en la focalización del servicio al cliente y en el diseño o rediseño de estrategias para la participación en el mercado, entre otros aspectos.

Finalmente, es sustancial reconocer las limitaciones del estudio y sugerir el interés de realizar nuevas investigaciones de carácter empírico, especialmente de estudios de casos, en profundidad, de tal forma que se pueda llegar a un conocimiento más detallado y realista de la situación de cada organización, en particular en aspectos relacionados con el rol y los determinantes de la innovación por diseño, así como también estudios con una muestra de empresas más amplia en diversas ciudades del país y, ojalá, en países con características similares que permitan un conocimiento más completo del tema en el contexto de la realidad empresarial para, con base en ello, diseñar estrategias que contribuyan a que las empresas hagan un mejor uso de la innovación por diseño como estrategia que garantiza la creación, de forma sostenida, de valor para sus diferentes grupos de interés.

\section{CONCLUSIONES}

En general, los resultados del estudio indican que los directivos entrevistados están de acuerdo en que la innovación es una variable estratégica para mejorar la competitividad empresarial. Sin embargo, las fuentes de sus innovaciones son en general sus propios planes y experiencias internas más que la información o el conocimiento proveniente de diferentes actores o agentes de la sociedad (instituciones académicas, centros de investigación y diseño, expertos en tendencias, etcétera) o del análisis de variables del entorno externo (asistencia a ferias y exposiciones, análisis de tendencias del mercado, tendencias tecnológicas, preocupación por el cuidado del medioambiente, etcétera).

En relación con la incorporación del concepto de innovación por diseño como estrategia para generar ventaja competitiva, este sólo tiende a emplearse para las actividades de marketing, realizar cambios o mejoras en sus bienes o servicios, cambiar o mejorar la imagen interna y externa de la empresa, lo cual evidencia la relación positiva entre la innovación por diseño y las actividades de marketing de la empresa pero no con la rentabilidad. De otra parte, en las empresas participantes del estudio, este concepto se asocia poco al cambio o mejora de la cultura organizacional y al potencial que tiene para contribuir, desde las diferentes actividades de la empresa, a mejorar la calidad de vida de sus grupos de interés y a proteger el medio ambiente. 


\section{REFERENCIAS}

Barrabés, C. Diseño y creación de conceptos empresariales innovadores. En: Jarauta, F. Cuadernos de diseño: Diseño, innovación y empresa. Madrid, Instituto Europeo di Design, 3, 167- 181 (2010)

Beacham, C. and Shambaugh, N. Contemporary Uses of Design Thinking Across Society, Work and the Individual. Design Principles and Practices: An International Journal, 5(5), 337-348 (2011)

Brown, T. Change by Design: How Design Thinking Transforms Organizations and Inspires Innovation. Harper Business, New York (2009)

Brunner, R. y Emery, S. El diseño sí importa, Folio Time Press, Nueva York (2008)

Comisión Europea. Aplicación de un plan de acción para la innovación en materia de diseño, [Translation Original document is in English, SWD 380] (2012)

Consejo Europeo de Liderazgo de Diseño. Diseño para el crecimiento y la prosperidad, informe y recomendaciones. https://goo.gl/w14WKL. Acceso: 9 de agosto (2013)

Chayutsahakij, P. and Poggenpohl, S. User-Centered Innovation: The Interplay between User-Research and Design Innovation. Proceedings of the European Academy of Management $2^{\text {nd }}$ Annual Conference on Innovative Research in Management, EURAM, Stockholm, Sweden (2002)

Costa, J. Diseño global en la empresa. En: Jarauta, F. Cuadernos de diseño: Diseño, innovación, empresa. Madrid, Instituto Europeo di Design, 3, 150- 165 (2010)

Cuesta, M. Diseño, innovación y empresa: Los tres pilares que conducen al éxito. En: Jarauta, F. Cuadernos de diseño: Diseño, innovación, empresa. Madrid, Instituto Europeo di Design, 3, 50 - 62 (2010)

Da Silva, D. N., Vieira, R. K., Vieira, A. K. y De Santiago, M. Optimización del Proceso de Innovación para Proyectos Internos en las Empresas, Información Tecnológica, 27(3), 119-130 (2016)

De Bevilacqua, C. Environmental innovation. En: Jarauta, F. Cuadernos de diseño: Diseño, innovación, empresa. Madrid, Instituto Europeo di Design, 3, 130- 146 (2010)

Dow, S. P., Glassco, A. y otros tres autores, Parallel prototyping leads to better design results, more divergence, and increased self-efficacy. ACM Trans. on Computer-Human Interaction, 17 (4), 18 (2010)

Espinosa, F. F., Días, A. y Back, N. Un Procedimiento de Evaluación de las Condiciones Necesarias para Innovar la Gestión de Mantenimiento en una Empresa, Información Tecnológica, 19(1), 97-104 (2008)

European Comission. Implementing an Action Plan for Design-Driven Innovation. European Comission, Bruselas (2013)

Gómez, Y. Innovación basada en el pensamiento y la estrategia de diseño. Revista académica e institucional de la UCPR, 84, 69-96 (2009)

Hollanders, H. y A., Van Cruysen. Design, Creativity and Innovation: A Scoreboard Approach. UNU-MERIT, Maastricht Economic and social Research Centre on Innov. and Technol. Maastricht Univ, Holland (2009)

Jaramillo, H., Jugones, G. y Salazar, M. Manual de Bogotá: Normalización de Indicadores de Innovación Tecnológica en América Latina y el Caribe. Ricyt, Colciencias, Cyted y Ocyt, Bogotá (2000)

John, H. Toothpicks \& Logos: Design in Everyday Life. Oxford University Press, New York (2002)

Johnson, M. y L.-S. McHattie. Making design explicit in organisational change: Detour o Latour, $19^{\text {th }}$ DMI: Academic Design Management Conference, London, 2- 4, September (2014)

Kilinc, L., Basak Ozturkb, G. and Yitmen, I. The changing role of the client in driving innovation for design build projects: stakeholders' perspective. Procedia Economics and Finance, 21, 279 - 287 (2015)

Kumar, V. y W.P. Faster, Deeper User Research. Design Management Journal, 14(2), 50-55 (2003)

Lecuona, M. Conceptos básicos de la gestión del diseño en las pymes, Edición Servicio de Publicaciones Universidad Politécnica de Valencia - UPV, Valencia (2005)

Leonar-Barton, D. Capacidades empresariales para la innovación y su gestión. Harvard Business School Press, Boston (2005)

Lojacono G. and Zaccai, G. The evolution of the Design-Inspired Enterprise. Sloan Management Review, Spring, 75-79 (2004)

Manzini, E. Una economía verde, social y en redes. En: Jarauta, F. Cuadernos de diseño: Diseño, innovación, empresa. Madrid: Istituto Europeo di Design., 3, 66-81 (2010) 
Mutlu, B. and Er, A. Design Innovation: Historical and Theoretical Perspectives on Product Innovation by Design: 5th European Academy of Design Conference held in Barcelona, Barcelona (2003)

Norman, D. and Verganti, R. Innovation and Design Research. Presented at Designing Pleasurable Products and Interfaces, (DPPI), JDNOR, Milan (2011)

Norman, D. and Verganti, R. Incremental and radical innovation: design research versus technology and meaning change. Politecnico di Milano and Mälardalen University, Milano (2012)

Norman, D. and Verganti, R. Incremental and Radical Innovation: Design Research vs. Technology and Meaning Change. MIT Press Journals, 30(1), 78-96 (2014)

NWRA and the CIRCA Group Europe. Design driven innovation: why it matters form SME competitiveness. Northern and Western Regional Assembly, Brussels (2015)

Perks, H., Cooper, R. y Jones, C. Characterizing the Role of Design in New Product Development: An Empirically Derived Taxonomy. J. Product Innovation Management, 22, 11-127 (2005)

Potier, O., Brunc, J., Le Massonc, P. and Weil, B. How innovative design can contribute to Chemical and Process Engineering development? Opening new innovation paths by applying the $\mathrm{C}-\mathrm{K}$ method Olivier. Chemical engineering research and Design, 103, 108-122 (2015)

Power, D. The Future in Design: The Competitiveness and Industrial Dynamics of the Nordic Design Industry. Uppsala, Centre for Research on Innovation and Industrial Dynamics, Sweden (2004)

Rivera, J. y Vidal, R. Valor de las metodologías de diseño en los procesos de gestión de la innovación. 3er Congreso Iberoamericano de Innovación Tecnológica, Universidad de Guadalajara, Guadalajara (2008)

Rodríguez, A. y Rodríguez, D. Política nacional de diseño. Chile. Consulting Design, Chile (2007)

Rosenthal, S.R. and Capper, M. Ethnographies in the Front End: Designing for enhanced Customer Experiences, Journal of Product Innovation Management, 23 (3), 215-237 (2006)

Santolaria, M., Oliver-Solà, J., y otros tres autores, Eco-design in innovation driven companies: perception, predictions and the main drivers of integration. The Spanish example. J. Cleaner Prod.,19,1315-1323 (2011)

Sandberg, B. Managing and Marketing Radical Innovations, Routledge, New York (2011)

Selden, Larry. Gestión de la innovación centrada en el cliente. Harvard Business Review, 27(33), 80 -88 (2006)

Soh, Pek-Hooi, Roberts, E. B. Networks of innovators: a longitudinal perspective. Research Policy, 32, 1569-1588 (2003)

Sorenson, O. and Waguespack, D.M. Research on social networks and the organization of research and development: an introductory essay. Journal of Engineering and Technology Management, 22, 1-7 (2005)

Ulrich, K. Product Design and Development, McGraw Hill, New York (2004)

Utterback, J., Vedin, B., Álvarez, E., Ekman, S., Sanderson, S., Tether, B. and Berganti, R. Design Inspired Innovation. World Scientific Publishing, New York (2006)

Veryzer, R. W. and Borja de Mozota, B. The Impact of User-Oriented Design on New Product Development: An Examination of Fundamental Relationship. Journal of Product Innovation Management, 22, 128-143 (2005)

Verganti, R. Design-driven innovation: changing the rules of competition by radically innovating what things mean. Harvard Business Press, Boston, M.A. (2009)

Verganti, R. User-Centered Innovation Is Not Sustainable, Harvard Business Review, Blogs (2010a)

Verganti, R. Design, meanings and radical innovation: A meta-model and a research agenda. Economics and Industrial Engineering, Politecnico di Milano, Milano (2010b)

Verganti, R. Design Driven Innovation: Changing the Rules of Competition by Radically Innovating What Things Mean, New York, McGraw Hill (2013)

Verganti, R. and Öberg. A. Interpreting and Envisioning: A Hermeneutics Approach to Radical Innovation. Industrial Marketing Management, 24(2), 37- 54 (2012)

Von Hippel, E. Usuarios y suministradores como fuentes de Innovación. Fundación Cotec para la innovación tecnológica, Madrid (2004)

Xu, F. y Rickards, T. Creative Management: A Predicted Development from Research into Creativity and Management. Creativity and Innovation management, 16(3), 216-228 (2000) 